Doug Geisler, Eva K. Grebel, and Dante Minniti, eds.

\title{
M31 Globular Cluster Metallicities and Ages
}

\author{
Pauline Barmby \\ Harvard-Smithsonian Center for Astrophysics, 60 Garden St., Mailstop \\ 10, Cambridge MA 02138, USA
}

\begin{abstract}
Globular cluster ages are more than just lower limits to the age of the universe; the distribution of ages constraints the timescales for galaxy formation and chemical evolution. Globular cluster populations with different metallicities have now been detected in many galaxies, and understanding how these populations formed requires knowing their relative ages. We examined the relative ages of the two M31 globular cluster populations using their color and luminosity distributions and found that the metal-rich clusters could be up to $50 \%$ younger than the metal-poor clusters. While a small delay in the formation of metal-rich clusters might be imposed by chemical enrichment timescales, a large age gap demands a more detailed explanation. I outline several possibilities and their promises and problems.
\end{abstract}

\section{Introduction}

Studies of the M31 globular cluster system are thoroughly reviewed by Jablonka (these proceedings). One of the most important discoveries about the M31 GCS is that, like that of the Milky Way, it contains two populations of globular clusters with different metallicities, kinematics, and spatial distributions. Many other galaxies' GCSs also show this bimodality, and it has been seized upon as an important key to GCS and galaxy history. An important question is whether the two populations of clusters have different ages.

Measuring ages for individual M31 GCs by fitting isochrones to their CMDs is not possible with existing technology, although it is part of the design reference mission for NGST (Rich \& Neill 1999). Age estimates using horizontal branch morphologies, as done by Sarajedini et al. (2000) for M33 clusters, is possible, although it relies on the (still controversial) assumption that age is the dominant 'second parameter'. We used a new catalog of integrated properties of M31 GCs and GC candidates (Barmby et al. 2000) to investigate the ages of the metalpoor and metal-rich M31 GCs. An important feature of this catalog is its use to determine the extinction of individual clusters, and thus their intrinsic colors and magnitudes.

\section{Cluster colors and SSP models}

The original idea of this work (Barmby \& Huchra 2000) was to compare the intrinsic colors of M31 and Milky Way globular clusters to the predictions of 
simple stellar population models. This is important not only for eliciting the properties of M31 GCs, but also for testing the models. GCs are among the simplest stellar populations known, and models which cannot reproduce their integrated colors will presumably have problems when used to study composite populations like galaxies.

The intrinsic colors of M31 globular clusters are taken from our catalog (Barmby et al. 2000); colors for Milky Way globulars are from the Harris (1996) catalog, augmented by infrared colors published in Brodie \& Huchra (1990). For this comparison, we used only clusters with measured spectroscopic metallicities and reddening $E(B-V)<0.5$, since uncertainty in the reddening, and hence the intrinsic colors, increases for large values of $E(B-V)$.

We compared the cluster colors to those of simple stellar populations from three sets of models: those of Worthey, Bruzual \& Charlot (both the Worthey and $\mathrm{B} \& \mathrm{C}$ models are the versions reported in Leitherer et al. 1996), and Kurth, Fritze-von Alvensleben, \& Fricke (1999). We used all the available model metallicities, and model ages of 8, 12, and 16 Gyr. Although model colors are tabulated in smaller age increments (typically $1 \mathrm{Gyr}$ ), we decided that it was more reasonable to use only a few typical ages than attempt to derive precise cluster ages from integrated colors.

The models generally fit the data very well, with offsets in the bluer colors likely due to model problems. However, the best-fit models for the metal-rich clusters have younger ages $(8$ or $12 \mathrm{Gyr}$ ) than those for the metal-poor clusters $(16 \mathrm{Gyr})$. Unless there is some systematic problem with metallicity in our data and/or the models, this means that the metal-rich clusters are younger than the metal-poor clusters in both M31 and the Milky Way.

\section{GCLF variation}

Many factors influence the total luminosity of a globular cluster, including mass, IMF, age, and metallicity. It is thus somewhat surprising that the distribution of integrated GC luminosities, the globular cluster luminosity function or GCLF, is so similar from galaxy to galaxy. In Barmby, Huchra, \& Brodie (2001), we computed the GCLF for various sub-samples of the M31 GCS. We used the intrinsic $V_{0}$ magnitudes for clusters with $V<18$ and careful corrections for incompleteness, and computed the GCLF parameters using the maximum likelihood method described in Secker \& Harris (1993).

The peak of the GCLF was brighter for clusters near the center of M31 than for more distant clusters. The GCLF difference found was comparable to theoretical predictions of cluster destruction effects (e.g., Ostriker \& Gnedin 1997). We also found a difference between the GCLFs of metal-rich and metalpoor clusters. The metal-rich clusters are brighter, not fainter as would be expected from the effects of metallicity on $M / L_{V}$ (Ashman, Conti, \& Zepf 1995). One way for the metal-rich clusters to be brighter than the metal-poor clusters is for them to be significantly younger. Comparisons with the population synthesis models mentioned above show that the luminosity difference we measured could be caused by a difference in age of about $55 \%$. 


\section{Summary}

Integrated colors and luminosities of M31 globular clusters provide photometric evidence that at least some of the metal-rich GCs in M31 are younger than the bulk of the population. Such an effect has previously been suggested to explain the ratio of dwarf to giant light found in the spectra of metal-rich M31 clusters by Tripicco (1989). The age differences our results imply are large - up to 8 Gyr. Chemical evolution models generally do not require this much time to enrich protocluster gas, so we need some other reason for the large age gap between populations. One possibility is that the metal-rich clusters in M31 formed in a merger (as in the picture of Ashman \& Zepf 1992), but any such merger would have to be gentle enough not to destroy the M31 disk. Another possibility is that the metal-poor clusters were formed before the galaxy itself, and the metal-rich clusters formed with the galaxy. If this was the case, one might expect to see similar age differences in the GC populations of other galaxies. Integrated broadband magnitudes and colors are clearly not the best way to age-date globular clusters, although they provide tantalizing hints in the case of the M31 GCs. More precise methods are needed for age-dating extragalactic globular clusters.

Acknowledgments. I thank the conference organizers for a stimulating meeting and for the financial assistance which enabled me to attend, and the Smithsonian Institution for financial support.

\section{References}

Ashman, K. M. \& Zepf, S. E. 1992, ApJ, 384, 50

Ashman, K. M., Conti, A. \& Zepf, S. E. 1995, AJ, 110, 1164

Barmby, P. \& Huchra, J. P. 2000, ApJ, 531, L29

Barmby, P., Huchra, J. P., \& Brodie, J. P. 2001, AJ, 121, 1482

Barmby, P., Huchra, J. P., Brodie, J. P., Forbes, D. A., Schroder, L. L. \& Grillmair, C. J. 2000, AJ, 119, 727

Brodie, J. P. \& Huchra, J. P. 1990, ApJ, 362, 503

Harris, W. 1996, AJ, 112, 1487

Kurth, O. M., Fritze-von Alvensleben, U. \& Fricke, K. J. 1999, A\&AS, 138, 19

Leitherer, C. et al. 1996, PASP, 108, 996

Ostriker, J. P. \& Gnedin, O. 1997, ApJ, 487, 667

Rich, R. M. \& Neill, J. D. 1999, BAAS, 194, 712

Sarajedini, A., Geisler, D., Schommer, R. \& Harding, P. 2000, AJ, 120, 2437

Secker, J. \& Harris, W. E. 1993, AJ, 105, 1358

Tripicco, M. J. 1989, AJ, 97, 735

\section{Discussion}

G. Piotto: It is not totally correct to say that the Rosenberg et al. (1999) results show a younger age; because the original sample extends only to $[\mathrm{Fe} / \mathrm{H}] \sim-0.7$ 
and second because that result is totally model dependent. Instead, an extension of the original sample (see Poster) seems to indicate a larger age dispersion for metal-rich clusters. It would be rather interesting to see if a similar trend is present also in the M31 globular cluster population.

P. Barmby: I agree the result we present for M31 cluster colors is also totally model dependent. It would be great to have an age distribution for M31 globulars, rather than approximate ages for groups of clusters - but doing that from integrated colors is a very dangerous game.

M. Fall: Isochrone fitting is a more direct method of determining ages than integrated photometry. For globlar clusters in the Milky Way, unlike those in M31, the data are available for both methods. Thus, one can test the photometric method of age-dating the M31 clusters by applying it in the same way to the Milky Way clusters and comparing the results with isochrone fitting. Have you done this?

P. Barmby: Yes, and we do find a younger age for the metal-rich Milky Way clusters (although the result is weaker than for the M31 clusters because there are fewer low-reddening metal-rich Milky Way clusters). This is somewhat worrisome since the CMDs do not give such a large age difference for Milky Way clusters. It may mean that there is a problem with the models rather than a true age difference (for both M31 and Milky Way clusters), but there could also be problems with the integrated colors of Milky Way clusters, which are difficult to measure.

J. Brodie: I just wanted to clarify that when deducing relative ages from the GCLF turnovers you find that the red GCs are younger than the blue GCs. When this same exercise is conducted in the Milky Way we find the opposite result.

P. Barmby: That's correct: the GCLF for Milky Way clusters does not show significant differences between metal-rich and metal-poor clusters (and we did not reanalyze the Milky Way GCLF in our work).

F. D'Antona: I would like to know how the model colors of the SSPs are calibrated as a function of metallicity.

G. Bruzual: If the models are "theoretical" we must remember that they may be biased, as they dramatically depend on the red giant branch colors, whose dependence on the metallicity is not well known (this in turn is related to the efficiency of turbulent convection).

P. Barmby: Yes, the model colors of SSPs are theoretical, except for solar metallicity which are empirically calibrated. This is true for Bruzual and Charlot; I believe it's also true for Worthey and Kurth et al. but I'm not $100 \%$ sure. 